\title{
Clinicomycological Study of Otomycosis
}

\author{
Mariraj Jeer* and N. Mallika \\ Govt ITI College Road, Amarkhed Layout, Raichur 584102, India \\ *Corresponding author
}

\section{Keywords}

Clinicomycological study, Otomycosis,

A.niger, A.terreus, Antifungal eardrops

Article Info

Accepted:

12 March 2019

Available Online:

10 April 2019

\section{A B S T R A C T}

To study the various fungi causing otomycosis with its isolation and identification of species from the clinical laboratory of Vijayanagara Institute of Medical Sciences, Ballari, ear swab samples are collected from the department of ENT which is suspected for fungal cause of otomycosis. $\mathrm{KOH}$ mount was done for the presence of fungal elements and also Grams staining of the sample is done to look for uniformly stained Gram positive fungal elements. Another ear swab from the same ear is directly streaked on the SDA slant for fungal culture. The tubes are incubated at 37 degree Celsius for 1 month. Intermittently the tubes are checked for fungal growth. A total of 60 samples were collected from January 2018 to June 2018 from suspected cases of otomycosis in Department of ENT. Maximum cases were isolated from age group between $11 \mathrm{y}-20 \mathrm{y}$ with higher incidence among males42 cases $(70 \%) .45$ cases are positive for $\mathrm{KOH}, 48$ cases were positive for fungal culture. The isolates are as follows: A. niger (33.3\%), A. flavus (31.6\%), A. terreus $(3.3 \%)$, others $(11.2 \%)$ and no growth were (20\%) From the above study, 11y-20y constitute the higher incidence of fungal infection in ear with male preponderance and the most common fungi isolated in otomycosis were A. niger followed by A. flavus. Early detection and treatment of otomycosis helps in preventing the invasion of infection further. Timely instillation of antifungal eardrops will help to subside the infection.

\section{Introduction}

Otomycosisis a common condition encountered in a general otolaryngology clinical setting among patients who presented with signs and symptoms of otitis externa. It is a pathologic entity, with candida and Aspergillus the most common fungal species $^{1,2}$.

It is not clear that the fungi are the true infective agents or mere colonization species as a result of compromised local host immunity secondary to bacterial infection. Various predisposing factors include a humid climate, presence of cerumen, instrumentation of the ear, increased use of topical antibiotics / steroid preparations, immunocompromised host, patients who have undergone open cavity mastoidectomy and those who wear hearing aids with occlusive ear mold ${ }^{3}$.The infection is usually unilateral and characterized by inflammatory pruritis, scaling and otalgia ${ }^{4}$. 


\section{Materials and Methods}

From the clinical laboratory of Vijayanagara Institute of Medical Sciences, Ballari, ear swab samples are collected from the department of ENT which are suspected for fungal cause of otomycosis. $\mathrm{KOH}$ mount was done for the presence of fungal elements and also Grams staining of the sample is done to look for uniformly stained Gram positive fungal elements. Another Ear swab from the same ear, swab is directly streaked on the SDA slant for fungal culture. The tubes are incubated at 37 degree Celsius for 1month. Intermittently the tubes are checked for fungal growth. Appropriate statistical analysis is done at the end of the results.

\section{Results and Discussion}

A total of 60 ear swabs are collected from the Department of ENT. The group consisted of 42 males $(70 \%)$ and 18 females (30\%). Most of the cases were out patients about 52cases $(86.6 \%)$ and in patients were 8 cases $(13.3 \%)$. The highest incidence of age group is $11 \mathrm{y}-20 \mathrm{y}$ $(35 \%)$ and least incidence was seen in age group of 31y-40y (1.6\%) and 71y-80y (1.6\%). Out of 60 samples $45(75 \%)$ samples showed $\mathrm{KOH}$ positive and $48(80 \%)$ samples showed SDA culture positive. The percentage of fungal isolates is as follows: A.niger-20 cases (33.3\%), A.flavus-19 cases (31.6\%), A.terreus-2 cases (3.3\%), Fusarium spp-1 case $(1.6 \%)$, Penicillium-1 case $(1.6 \%)$, Geomyces destructans-1 case (1.6\%), Bipolaris-1 case (1.6\%), Mucor-1 case (1.6\%), Rhizopus-1 case (1.6\%), Candida-1 case (1.6\%) and NO GROWTH-12 cases $(20 \%)$.

Otomycosis is a superficial mycotic infection of the outer ear canal frequently encountered by otolaryngologist and can usually be diagnosed by clinical examination. However the correct diagnosis requires a high index of suspicion. The infection may be either sub acute or acute and is characterized by inflammation, pruritis, scaling and severe discomfort. The mycosis results in inflammation, superficial epithelial masses of debris containing hyphae, suppuration and pain ${ }^{4}$. In a study conducted by Rajeshwari Prabhakar Rao et al., shows higher incidence of otomycosis in the age group of $21 \mathrm{y}-30 \mathrm{y}$ (34\%) followed by $11 \mathrm{y}-20 \mathrm{y}(23.4 \%)$ but in our study the higher incidence of age is seen in $11 \mathrm{y}-20 \mathrm{y}(35 \%)^{5}$ as shown in Figure 1.

In our study the males (42cases-70\%) are affected more than females(18cases-30\%). Similar observations were seen in the study conducted by Zaror et al., 45 cases out of 60 were positive for $\mathrm{KOH}$ mount and 48 cases $(80 \%)$ were positive fungal culture. Aspergillus niger (20cases-33.3\%), Aspergillus flavus (19 cases-31.6\%) showed highest fungal isolates. These results were similar to the study done by Favour Osazuwa et al., ${ }^{5,7}$. Other fungal isolates in this study are as follows-A.terreus-2cases (3.3\%), Fusarium spp-1 case (1.6\%), Penicillium-1 case (1.6\%), Geomyces destructans-1 case (1.6\%), Bipolaris-1case (1.6\%), Mucor-1 case (1.6\%), Rhizopus-1 case (1.6\%), Candida-1 case $(1.6 \%)$ and NO GROWTH-12 cases $(20 \%)$. These observations were similar to that of the study done by Rajeshwari Prabhakar Rao et al., showing Candida (8.5\%), Mucor (2.1\%) and Penicillium $(2.1 \%)^{5}$ as shown in Figure 2.

If the fungal infection is not treated timely it leads to complications such as tympanic membrane perforation, hearing loss and invasive temporal bone infection ${ }^{8}$.

Treatment involves elimination of predisposing factors. Topical antibiotic solutions must be stopped. Patients' nails must be inspected to rule out onychomycosis. The ear canal must be thoroughly debrided of 
all visible debris. It is our practice to avoid syringing and clear the debris by suctioning alone. Fungicidal drops are the most popular form of treatment. Clotrimazole has an antibacterial effect, and this is an added advantage when treating mixed bacterialfungal infections. Fungicidal creams with ketoconazole or fluconazole may also be applied ${ }^{9}$.

Fig.1 Age wise distribution

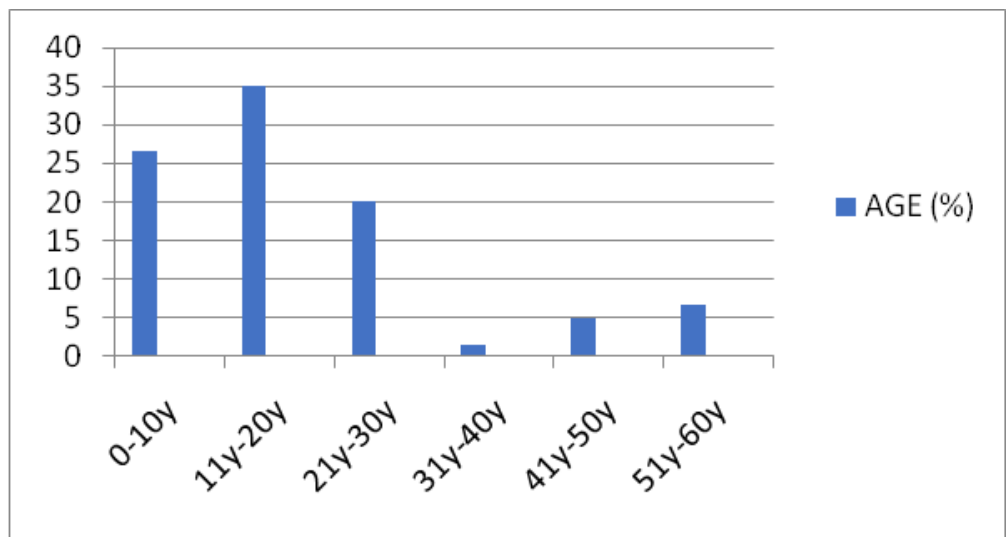

Fig.2

\section{Fig 2 showing no of isolates (\%)}

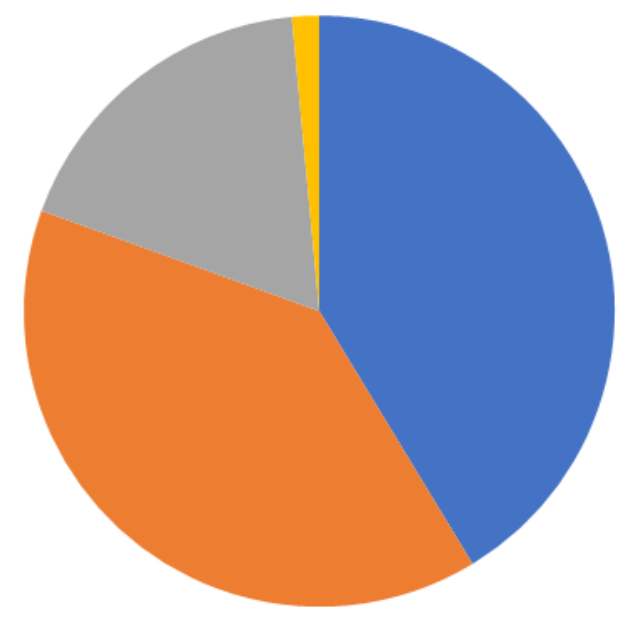

A. Niger

A. Flavus

others

No growth

Another technique in which the absorbable gelatin sponge is used for the treatment of recurrent and persistent otomycosis ${ }^{10}$. In our hospital because of inadequate resources, antifungal susceptibility testing is not done. But with the antifungal susceptibility appropriate antifungals are advised which will help accurate treatment of otomycosis. In conclusion, from the above study it is clear that clinical suspicion of otomycosis can prevent unnecessary use of antibiotics. High incidence of Otomycosis is reported in tropical countries. In our study, Aspergillus species was the commonest fungi involved in 
Otomycosis. As clinical features are non specific, laboratory diagnosis helps to know the exact etiology of Otomycosis to initiate appropriate antifungal therapy. Appropriate antifungals are advised based on antifungal susceptibility pattern and accurate treatment is started for better prognosis. Educating the rural population is another important concern and needs to be addressed.

\section{References}

1. Mugliston T, O'Donoghue G. Otomycosis: A continuing problem. J Laryngol Otol. 1985; 99(4): 327-333.

2. Kaur R, Mittal N, Kakkar M, Aggarwal AK, Mathur MD. Otomycosis; a clinicomycologic study. Ear Nose Throat J. 2000; 79(8): 606-960.

3. Vennevald I, Schonlebe J, Klemm E. Mycological and histological investigations in Humans with middle ear infections. Mycoses. 2003; 46 (1-2): 1218.

4. Anwar K, Gohar MS. Otomycosis; clinical features, predisposing factors and treatment implications. Pak J Med Sci 2014; 30(3): 564-567.

5. RajeshwariPrabhakar Rao, Rishmitha Rao. A mycologic study of otomycosis in a tertiary care teaching hospital in
Karnataka, India. International Journal of Contemporary Medical Research 2016;3(7):1918-1920.

6. Zaror L, Fischman O, Suzuki FA, Felipe RG. Otomycosis in São Paulo. Rev Inst Med Trop São Paulo. 1991;33:16973.

7. Osazuwa F, Osazuwa E, Osime C, Igharo EA, Imade PE, Lofor P, Momoh M, Omoregie R, Dirisu J. Aetiologic agents of otitis media in Benin city, Nigeria. North Am J Med Sci 2011; 3: 95-98.

8. Borlingegowda Viswanatha and Khaja Naseeruddin. Fungal Infections of the Ear in immunocompromised host: Review. Medit J Hemat Infect Dis 2011, 3: e2011003.

9. Sampath Chandra Prasad, Subbannayya Kotigadde, Manisha Shekhar, Nikhil Dinaker Thada, Prashanth Prabhu, Tina D'Souza, and Kishore Chandra Prasad. Primary Otomycosis in the Indian Subcontinent: Predisposing Factors, Microbiology, and Classification. International Journal of Microbiology Volume 2014, Article ID 636493, 1-9.

10. Surya Prakash Dorasala, Srinivas Dorasala. Medicated gelfoam for the treatment of recalcitrant otomycosis. Indian Journal of Otology. January 2013, Vol 19, Issue 1:18-19.

\section{How to cite this article:}

Mariraj Jeer and Mallika, N. 2019. Clinicomycological Study of Otomycosis. Int.J.Curr.Microbiol.App.Sci. 8(04): 1334-1337. doi: https://doi.org/10.20546/ijcmas.2019.804.155 